\title{
Changes in gonadotrophin-releasing hormone (GnRH-I) in the pre-optic area and median eminence of starlings (Sturnus vulgaris) during the recovery of photosensitivity and during photostimulation
}

\author{
A. Dawson ${ }^{1}$ and A. R. Goldsmith ${ }^{2}$ \\ ${ }^{I}$ Institute of Terrestrial Ecology, Monks Wood, Abbots Ripton, Huntingdon, Cambs., PE17 2LS, UK; and \\ ${ }^{2}$ School of Biological Sciences, University of Bristol, Woodland Road, Bristol, BS8 IUG, UK
}

\begin{abstract}
Changes in GnRH-I in the pre-optic (POA) and medio-basal (MBH) areas of the hypothalamus and in pituitary and plasma $\mathrm{LH}$ were measured in starlings (Sturnus vulgaris) during the recovery of photosensitivity under short days, and following photostimulation at various times during the recovery of photosensitivity. During exposure to short days there was a significant increase in GnRH-I in the POA, with the first detectable increase after only 10 days. There was no increase in GnRH-I in the MBH or in pituitary or plasma LH. In birds photostimulated after 10 short days, there was an increase in GnRH-I in the POA, but this was no greater than that in birds remaining under short days. There was no increase in GnRH-I in the MBH or in plasma LH. Photostimulation after 20 short days caused an immediate increase in GnRH-I in the POA, a delayed increase in GnRH-I in the MBH, but no increase in plasma LH. Photostimulation after 30 short days caused an immediate increase in GnRH-I in the POA and the MBH and in plasma LH. The results show that the recovery of photosensitivity is gradual; the first measurable change occurs in the POA, consistent with photosensitivity being due to renewed GnRH-I synthesis. The effects of photostimulation increase, both in magnitude and in terms of how far 'downstream' of the POA changes are apparent, as photosensitivity is gradually restored. The results support the hypothesis that daylength has a dual role, controlling both synthesis and secretion of $\mathrm{GnRH}$.
\end{abstract}

\section{Introduction}

In passerine birds, gonadal maturation at the beginning of the breeding season is stimulated by the vernal increase in daylength. Gonadal regression, at the end of the breeding season, is caused, not by a decrease in daylength, but by the development of photorefractoriness during long days. Termination of photorefractoriness (the recovery of photosensitivity) occurs during short days (for reviews see Nicholls et al., 1988; Wilson and Donham, 1988; Cockrem, 1995; Sharp, 1996).

The hypothalamo-pituitary-gonadal axis differs considerably between these two states. In photorefractory birds the amount of gonadotrophin-releasing hormone $(\mathrm{GnRH})$ in the hypothalamus is low, as judged by radioimmunoassay and immunocytochemistry (Dawson et al., 1985; Dawson et al., 1986; Foster et al., 1987; Bluhm et al., 1991; Saldanha et al., 1994; Hahn and Ball, 1995). Plasma gonadotrophin concentrations are low and are unaffected by gonadal steroids (Goldsmith and Nicholls, 1984; Wilson, 1985) or an increase in daylength. In photosensitive birds, hypothalamic GnRH is high. Although plasma concentrations of gonadotrophins remain low under short days, they are sensitive to steroid feedback; gonadectomy results in an increase in gonadotrophins. An increase in daylength stimulates an

Received 17 March 1997. increase in gonadotrophin secretion and consequent gonadal maturation.

Long days have two effects. One effect is gonadostimulatory, inducing gonadal maturation in photosensitive birds; the other is gonado-inhibitory, in that long days induce and maintain photorefractoriness. One possible explanation of this apparent contradiction is that daylength has two separate effects on GnRH neurones (Dawson et al., 1988). The first is an effect on GnRH release from the median eminence: the longer the daylength, the greater the release rate so that circulating gonadotrophin concentrations increase. The second effect of daylength is on GnRH synthesis: chronic exposure to long days downregulates synthesis (photorefractoriness) and, conversely, exposure to short days causes it to resume (photosensitivity).

In starlings, the recovery of photosensitivity, as judged by gonadal growth following photostimulation or by the increase in plasma gonadotrophins in gonadectomized birds, becomes apparent about 4 weeks after transfer to short days (Dawson, 1991; Boulakoud and Goldsmith, 1994; Boulakoud and Goldsmith, 1995). The first aim of this study was to examine changes in hypothalamic $\mathrm{GnRH}$ during this 4 week lag period. Does an increase in GnRH synthesis precede the recovery of photosensitivity? Is the increase first detectable in the cell bodies, suggesting that this is due to renewed synthesis rather than decreased release? 
The second aim was to compare the changes in GnRH synthesis and secretion during the recovery of photosensitivity with those during photostimulation. These changes cannot be assessed directly. Instead, we attempted to assess them indirectly by measuring the variation with time in the amounts of $\mathrm{GnRH}$ in different regions of the hypothalamus and the associated effect on gonadotrophin secretion. Even this is not possible during photostimulation of fully photosensitive birds. Since plasma concentrations of LH and FSH increase markedly following photostimulation, an increase in secretion of $\mathrm{GnRH}$ can be inferred. As there is comparatively little increase in total hypothalamic GnRH (Dawson et al., 1985), the increase in secretion must be matched by an increase in the rate of synthesis. Changes in the quantitative distribution of GnRH in this situation would not have revealed any information about the dynamics. However, since the recovery of photosensitivity appears to be gradual (Boulakoud and Goldsmith, 1995), it is possible that synthesis and storage of $\mathrm{GnRH}$ increases gradually during this recovery. Photostimulation during this period may result in variations in quantitative distribution, from which the changes in GnRH synthesis and secretion induced by long days could be assessed. The effects of photostimulation were therefore assessed during the recovery of photosensitivity.

\section{Materials and Methods}

\section{Birds}

Juvenile female starlings were caught from the wild during the summer. At this time they can be distinguished from adults by their plumage. (Moult from the light brown juvenile plumage into dark adult plumage is completed by early October.) They were kept in outdoor aviaries until the following January when they were moved indoors and kept in groups of four or five birds per cage. They were provided with food (chick starter crumbs) and water ad libitum. Initial daylength was $8 \mathrm{~h}$ light per day (8L:16D) and this was increased after two weeks to $18 \mathrm{~L}: 6 \mathrm{D}$. The experiment was started 6 months later, by which time all birds would have completed a cycle of gonadal growth and regression, become fully photorefractory, and completed their post-nuptial moult.

\section{Experimental protocol}

Birds were divided into ten groups of six. Group 1 birds were sampled (see below) after 6 months on 18L:6D and the remaining groups were transferred to short days $(8 \mathrm{~L}: 16 \mathrm{D}$; lights on 08:00 h, off 16:00 h). Ten days later, birds in group 2 were sampled, and at the same time groups 3 and 4 were moved to 18L:6D (lights on 08:00 h, off 02:00 h) and sampled 5 or 10 days later, respectively. This protocol was repeated after 20 short days (for groups 5, 6 and 7) and after 30 short days (for groups 8, 9 and 10), so that group 10, for example, had experienced 30 short days followed by 10 long days (Fig. 1). All birds were sampled between 10:00 h and 12:00 h.

\section{Sampling procedure}

Each bird was decapitated and trunk blood was collected into a heparinized tube. This was later centrifuged at $1000 \mathrm{~g}$ for

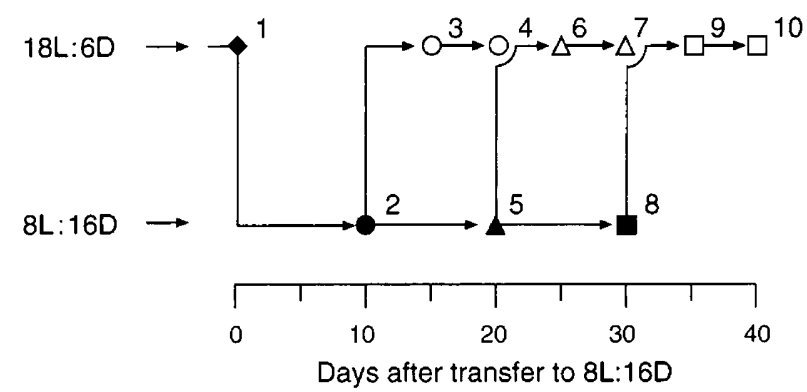

Fig. 1. Experimental protocol: photorefractory female starlings under a photoperiod of $18 \mathrm{~h}$ light: $6 \mathrm{~h}$ dark (18L:6D) were divided into ten groups $(n=6)$. Birds in group $I$ were sampled on day 0 . The remaining groups were transferred to $8 \mathrm{~L}: 16 \mathrm{D}$. Ten days later, birds in group 2 were sampled, and at the same time groups 3 and 4 were moved to $18 \mathrm{~L}: 6 \mathrm{D}$ and sampled 5 or 10 days later, respectively. This protocol was repeated after 20 short days (for groups 5,6 and 7) and after 30 short days (for groups 8,9 and 10). The symbols correspond to those in Fig. 2.

$10 \mathrm{~min}$ and the plasma was stored at $-70^{\circ} \mathrm{C}$. The head was dissected and bone removed from the base of the brain. The pituitary gland was removed, and then parasagittal cuts were made approximately $2 \mathrm{~mm}$ either side of the midline from the pre-optic area (POA) posteriorly to the habenular region. The resulting block of tissue was removed and cut into two with a horizontal cut in line with the dorsal surface of the optic chiasma (see Foster et al., 1987 for anatomy and distribution of GnRH cells in starlings). The upper block (POA) thus contained the GnRH perikarya and approximately one third to one half of the length of the fibre tracts, whereas the lower block, the medio-basal hypothalamus $(\mathrm{MBH})$, contained the remaining length of fibre tracts and the median eminence. A similar sized block of tissue was removed anterior to the POA to act as a control. The whole dissection procedure took 3-4 min.

One potential problem with this approach is contamination of one tissue block with GnRH from the other, the major risk being contamination of the POA with GnRH from the median eminence, where much greater amounts are stored. The division between the two blocks was made nearer to the cell bodies than the median eminence to minimise this risk. Significant cross-contamination may have been revealed as measurable $\mathrm{GnRH}$ in the control tissue blocks taken anterior to the POA. GnRH was not detectable in these samples.

The two hypothalamic tissue blocks and the control tissue were immediately homogenized in $2 \mathrm{ml}$ ice-cold $2 \mathrm{~mol}$ acetic acid $l^{-1}$. The homogenates were centrifuged at $7000 \mathrm{~g}$ for $1 \mathrm{~h}$. The supernatants were aspirated, lyophilized, and the residue stored at $-70^{\circ} \mathrm{C}$ until assay. The pituitary was homogenized in $400 \mu \mathrm{l}$ ice-cold 0.2 mol ammonium hydrogen carbonate $1^{-1}$ and centrifuged at $7000 \mathrm{~g}$ for $\mathrm{I}$ h. The supernatant was aspirated and frozen until assayed.

\section{Radioimmunoassays}

In birds, two different forms of GnRH have been identified. Only GnRH-I is thought to have a role in reproduction (Sharp et al., 1990) and consequently GnRH-l specifically was measured in this study. Concentrations of GnRH-I in the POA 

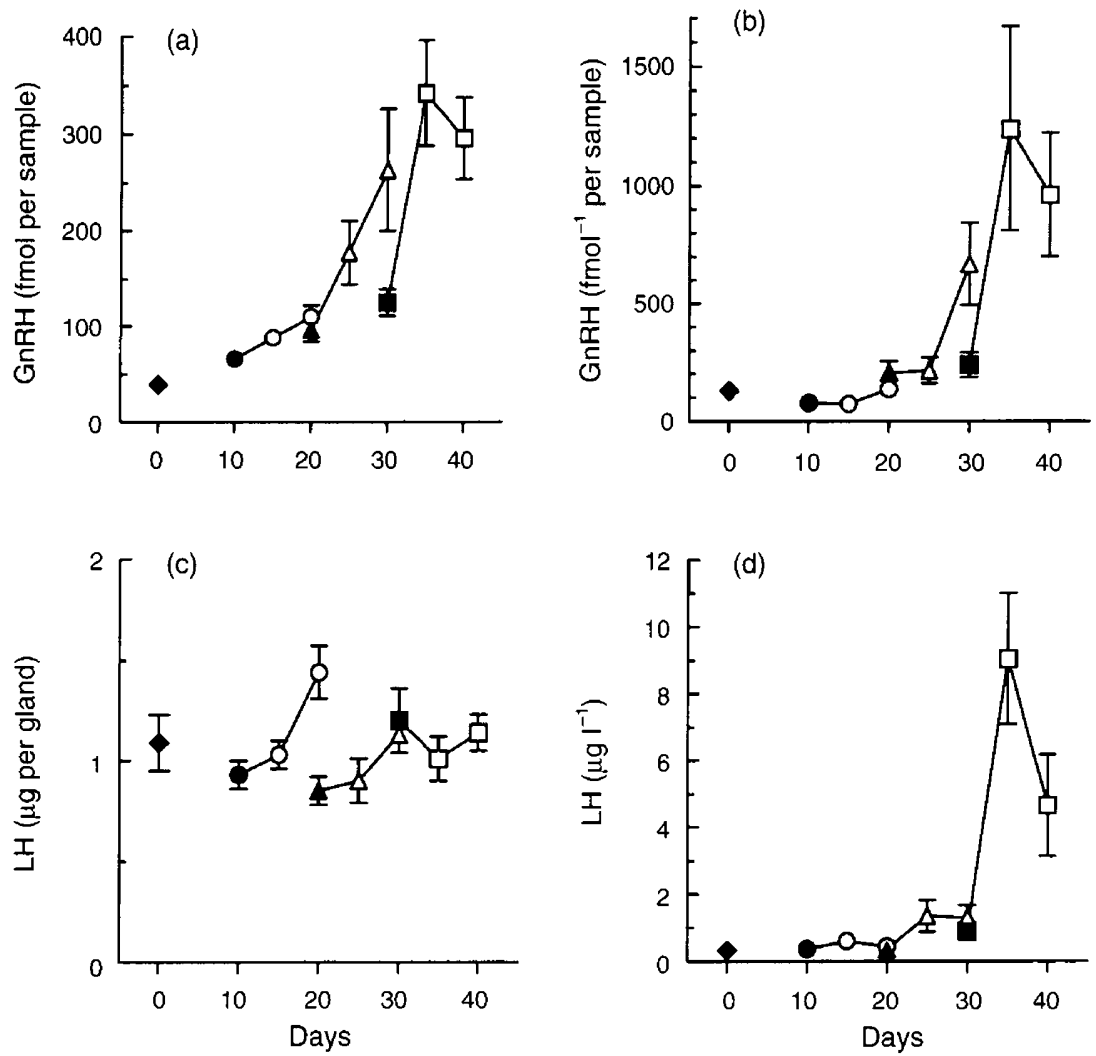

Fig. 2. Changes in gonadotrophin-releasing hormone ( $\mathrm{GnRH}-\mathrm{I})$ in the (a) pre-optic area (POA) and (b) medio-basal hypothalamus (MBH) and in (c) pituitary and (d) plasma LH during exposure to short days (solid symbols) and after transfer to long days after 10 short days (circles), 20 short days (triangles) or 30 short days (squares). Each point represents the mean \pm SEM for six birds.

and $\mathrm{MBH}$ extracts were determined using a homologous radioimmunoassay for chicken GnRH-I (Sharp et al., 1990) as used in starlings (Dawson and Goldsmith, 1989; Goldsmith et al., 1989). An antiserum raised against chicken GnRH-I (code $3 / 3$, supplied by P. J. Sharp, Roslin Institute, Midlothian) was used at a final concentration of 1:18 000 with iodinated synthetic GnRH-I. The peptide (Peninsula Laboratories, St Helen's, Merseyside) was labelled with ${ }^{125}$ I by the chloramine-T method as described by Sharp et al. (1990). The assay is specific for chicken GnRH-I, showing negligible crossreaction with chicken GnRH-II or with ovine GnRH (Goldsmith et al., 1989; Sharp et al., 1990). Starling hypothalamic extracts dilute parallel to the chicken GnRH-I standard curve, and two starling extract pools yielded intra-assay coefficients of variation of $3.9 \%(n=5)$ and $6.3 \%(n=6)$, and inter-assay coefficients of variation of $5.2 \%(n=12)$ and $8.4 \%$ $(n=12)$. The lyophilized starling POA and MBH extracts were reconstituted in $1 \mathrm{ml}$ assay diluent and then assayed in duplicate at three dilutions in a single assay run. The sensitivity of the assay was $5 \mathrm{fmol}$ per sample. The concentrations of $\mathrm{GnRH}-\mathrm{I}$ in the anterior brain sections were below this detection limit.

For the LH assay, pituitary extracts were diluted 500 -fold in assay diluent. Aliquots, $20 \mu \mathrm{l}$, of the diluted extracts and $20 \mu \mathrm{l}$ aliquots of plasma were assayed in duplicate using a homologous chicken LH radioimmunoassay (Follett et al., 1972; Follett et al., 1975; Dawson and Goldsmith, 1982). The antiserum was anti-IRC2T at 1:6000. The sensitivity of the assay was $0.1 \mu \mathrm{g}$ chicken $\mathrm{LH} \mathrm{l}^{-1}$ plasma and $0.2 \mu \mathrm{g} \mathrm{LH}$ per pituitary gland.

\section{Statistical analyses}

Data were normalized by $\log$ transformation before analysis. Groups 1, 2, 5 and 8 were compared, to assess changes during exposure to short days, using single factor analysis of variance (ANOVA) followed, where significant, by Dunnet's Multiple Comparisons, comparing values following transfer to short days with the value before transfer. Groups 2, 3 and 4; 5, 6 and 7; and 8,9 and 10 were treated separately to assess changes after transfer to long days after 10,20 or 30 short days respectively. Again, single factor ANOVA was used followed by Dunnet's Multiple Comparisons, comparing values following transfer to long days with the value before transfer.

\section{Results}

POA

There was a significant increase in GnRH-I in the POA during exposure to short days $(F(3,20)=19.1 ; P<0.0001)$ (Fig. 2). GnRH-I increased by $56 \%$ after only 10 short days $(P<0.05)$ and by $133 \%$ after 20 short days $(P<0.001)$. In birds 
transferred to long days after 10 short days, GnRH-I increased significantly $(F(2,15)=8.4 ; P=0.0036)$. Values had increased (but not significantly) after 5 days, and by 10 days had increased by $66 \%(P<0.01)$. However, interpretation of this increase is difficult because this was similar to the increase in birds remaining on short days over the same period. Thus this increase may have been entirely due to the effects of long days, a delayed effect of short days, or a mixture of the two. In birds transferred to long days after 20 short days, there was a significant increase $(F(2,15)=7.0 ; P=0.007)$ over and above that which occurred under short days over the same period. Values increased by $90 \%$ (not significant) after 5 days and $184 \%(P<0.01)$ after 10 days. In the case of birds transferred to long days after 30 short days $(F(2,15)=17.8 ; P<0.0001)$, values had doubled $(P<0.001)$ after only 5 days. Some caution is required here because there was no control group kept on short days beyond day 30. It is probable that $\mathrm{GnRH}$ would also have increased under short days, and the magnitude of this increase is unknown. Nevertheless, the response to long days was greater and more rapid after 30 days than after 20 days, which itself was greater than that after 10 days.

\section{$\mathrm{MBH}$}

Changes in GnRH-I in the MBH showed a different pattern from those in the POA. GnRH-I did not change significantly during the 30 short days. After transfer to long days after 10 or 20 short days, there were marginally significant changes in GnRH-I $(F(2,15)=5.9(P<0.013)$ and $4.6(P<0.029)$, respectively). In contrast to changes in the POA, values in the $\mathrm{MBH}$ had not increased after 5 days. They had increased by 10 days $(P<0.05$ in each case), only slightly in birds transferred after 10 days, but more substantially in those transferred after 20 days. In birds transferred after 30 short days, GnRH-I changed markedly $(F(2,15)=8.5(P<0.004))$ with a significant increase $(P<0.01)$ after only 5 days.

\section{Pituitary $L H$}

There were no significant changes in pituitary LH during exposure to short days. There was an increase following transfer to long days after 10 short days $(F(2,15)=13.8)$ with values increasing significantly $(P<0.01)$ after 10 days. Transfer after 20 or 30 short days did not result in significant changes.

\section{Plasma LH}

There was no significant change in plasma LH during short days. Nor did LH change significantly in birds transferred to long days after 10 short days. There was a marginally significant increase following transfer after 20 short days $(F(2,15)=5.75 ; P=0.014)$ with concentrations after 5 and 10 days slightly higher $(P<0.05)$ than at the time of transfer. In birds transferred after 30 short days, changes were highly significant $(F(2,15)=14.58 ; P<0.001)$, with LH increasing 10-fold after 5 days $(P<0.001)$.

\section{Discussion}

In photorefractory birds at the beginning of this experiment, GnRH-I was low in both the POA and the MBH, and plasma
LH was low. Transfer to short days caused a significant increase in GnRH-I in the POA but not in the MBH. This suggests that the first changes associated with the recovery of photosensitivity occur in the GnRH-I perikarya rather than the median eminence. This could be a change in the rate of gene transcription or translation. It probably will not represent a change in the processing of pre-pro-GnRH to GnRH-I, because the GnRH-I antibody used in this study also recognizes the unprocessed peptide. This is consistent with the hypothesis that the recovery of photosensitivity is associated with renewed or upregulated $\mathrm{GnRH}$ synthesis. Although $\mathrm{GnRH}$ in the $\mathrm{MBH}$ did not increase under short days within the time course of this experiment, release of $\mathrm{GnRH}-\mathrm{I}$ from the median eminence must increase at some time under short days because pituitary stores of FSH do eventually increase (Dawson et al., 1986).

The increase in GnRH-I in the POA was significant after only 10 short days. In earlier studies, the first measurable change signalling the recovery of photosensitivity (spontaneous increase in plasma $\mathrm{LH}$ in gonadectomized birds or an increase in testicular size following photostimulation) occurred after about 4 weeks of short days (Goldsmith and Nicholls, 1984; Dawson, 1991; Boulakoud and Goldsmith, 1994; Boulakoud and Goldsmith, 1995). Clearly changes occur in the POA before more peripheral effects become apparent. In fact, since $\mathrm{GnRH}$ in the POA had increased significantly by 10 days, it is possible that the recovery of photosensitivity (upregulated GnRH synthesis) begins immediately after transfer to short days. The 4 week delay before the apparent return of photosensitivity may simply be the time taken for GnRH synthesis to increase sufficiently for subsequent photostimulation to induce a measurable effect.

In contrast to the effects of short days, photostimulation did cause effects 'downstream' from the POA. In birds transferred to long days after 20 short days, there was an increase in GnRH-I in the MBH. At the same time, GnRH-I in the POA increased over and above that caused by exposure to short days. Plasma LH also increased, but only in birds photostimulated after 30 short days. This finding is consistent with the hypothesis that increased daylength stimulates increased release of $\mathrm{GnRH}$ with a concomitant increase in the rate of synthesis.

The increase in GnRH-I in the POA preceded that in the $\mathrm{MBH}$ which in turn preceded an increase in plasma $\mathrm{LH}$. However, one surprising result was that pituitary LH content increased in photostimulated birds before a marked increase in $\mathrm{MBH}$ GnRH-I. Normally pituitary LH content in starlings remains fairly constant during both photostimulation and the onset of photorefractoriness (presumably GnRH-I stimulates synthesis and release equally). It is possible that in this experimental situation there was a brief period during which secretion of GnRH-I was sufficient to stimulate synthesis of $\mathrm{LH}$ but not its release, causing a build up of stored LH. Photostimulation later, which did result in LH secretion, did not alter stored LH.

The results confirm earlier evidence that the recovery of photosensitivity is gradual. GnRH in the POA increased steadily with exposure to short days and the effects of photostimulation increased the longer birds had been exposed to short days. In birds photostimulated after 10 short days 
there was an increase in GnRH-I in the POA, but this was no greater than in birds that remained on short days. After 20 days there was a marked increase, and an even more rapid increase after 30 days. There was no increase in GnRH in the MBH in birds photostimulated after 10 short days. There was a delayed increase after 20 days and after 30 days there was a rapid increase. There was no increase in plasma LH in birds photostimulated after 10 or 20 short days but there was after 30 days. Even after 30 days, photosensitivity was probably not fully restored; starlings photostimulated after 4 weeks of short days showed less testicular growth and more rapid regression than in birds photostimulated after 6 weeks (Boulakoud and Goldsmith, 1995).

In chronically photosensitive starlings, photostimulation resulted only in a slight and non-significant increase in total hypothalamic GnRH content (Dawson et al., 1985). Similarly, in Japanese quail (Coturnix coturnix japonica), photostimulation resulted in a large increase in plasma $\mathrm{LH}$, but no change in hypothalamic GnRH content (Creighton and Follett, 1987), which suggests that photostimulation causes an increase in GnRH secretion and a concomitant increase in synthesis. In this study photostimulation did result in an increase in GnRH, presumably because these birds were not fully photosensitive and GnRH synthesis and storage had not reached the maximum values eventually attained under short days. Indeed, this was the aim of the experimental design. Since photosensitivity increases in starlings until at least 6 weeks of short days, presumably $\mathrm{GnRH}$ would have increased under short days beyond the concentrations measured after 30 days.

The hypothesis is that recovery of photosensitivity is equivalent to renewed, or at least a marked increase in, GnRH-I synthesis, or more correctly pre-pro-GnRH synthesis. That the first discernible change in GnRH-I was an increase in the POA supports this (this would not be the case if photorefractoriness was an inhibition of release of GnRH-I). The corollary of this is that at the onset of photorefractoriness a decrease in hypothalamic GnRH-I should precede a decrease in circulating LH and gonadal regression. This is not the case; a measurable decrease in GnRH occurs only after gonadal regression (Dawson et al., 1985). This suggests, contrary to the hypothesis, that photorefractoriness is not a cessation of GnRH-I synthesis, but rather an inhibition of release of GnRH-I, which only later feeds back within the GnRH-I neurones to inhibit synthesis. However, it has been shown that the unprocessed peptide, pre-pro-GnRH, does decrease as gonadal regression occurs (Parry et al., 1997). Synthesis may therefore cease, and the persistent high $\mathrm{GnRH}$ concentrations may represent a residual unreleased pool. Measurement of pre-pro-GnRH mRNA could resolve this issue, but to date, attempts to clone the gene have not been successful in any passerine.

In conclusion, the data support the hypothesis that daylength has a dual role in the control of $\mathrm{GnRH}$, an effect on synthesis and on release. Under short days GnRH increased in the cell bodies, suggesting increased synthesis. After subsequent transfer to long days there was an increase in the median eminence and an increase in LH, suggesting increased secretion.

The authors are indebted to L. M. Glennie of the Department of Zoology, University of Bristol for assaying GnRH-I.

\section{References}

Bluhm CK, Schwabl H, Schwabl A, Perera A, Follett BK, Goldsmith AR and Gwinner, E (1991) Variation in hypothalamic gonadotrophin-releasing hormone content, plasma and pituitary $\mathrm{LH}$, and in-vitro testosterone release in a long-distance migratory bird, the garden warbler (Sylvia borin), under constant photoperiods Journal of Endocrinology 128 339-345

Boulakoud MS and Goldsmith AR (1994) Acquisition of photosensitivity in castrated male starlings (Sturnus vulgaris) under short day photoperiods Journal of Reproduction and Fertility 100 77-79

Boulakoud MS and Goldsmith AR (1995) The effect of duration of exposure to short days on the gonadal response to long days in male starlings (Sturnus vulgaris) Journal of Reproduction and Fertility 104 215-217

Cockrem JF (1995) Timing of seasonal breeding in birds, with particular reference to New Zealand birds Reproduction, Fertility and Development 7 1-19

Creighton JA and Follett BK (1987) Changes in gonadotrophin-releasing hormone and $\mathrm{LH}$ in Japanese quail during the first few days of photostimulation Journal of Endocrinology 113 419-422

Dawson A (1991) Effect of daylength on the rate of recovery of photosensitivity in male starlings (Sturnus vulgaris) Journal of Reproduction and Fertility 93 521-524

Dawson A and Goldsmith AR (1982) Prolactin and gonadotrophin secretion in wild starlings (Sturnus vulgaris) during the annual cycle and in relation to nesting, incubation and rearing young General and Comparative Endocrinology $48213-221$

Dawson A and Goldsmith AR (1989) Sexual maturation in starlings raised on long or short days: changes in hypothalamic gonadotrophin-releasing hormone and plasma LH concentrations Journal of Endocrinology 123 189-196

Dawson A, Follett BK, Goldsmith AR and Nicholls TJ (1985) Hypothalamic gonadotrophin-releasing hormone and pituitary and plasma FSH and prolactin during photostimulation and photorefractoriness in intact and thyroidectomized starlings (Sturnus vulgaris) Journal of Endocrinology 105 $71-77$

Dawson A, Goldsmith AR, Nicholls TJ and Follett BK (1986) Endocrine changes associated with the termination of photorefractoriness by short daylengths and thyroidectomy in starlings (Sturnus vulgaris) Journal of Endocrinology 110 73-79

Dawson A, Goldsmith AR, Nicholls TJ and Follett BK (1988) Comparative endocrinology of photorefractoriness In Acta XIX Congressus Internationalis Ornithologica pp 634-640 Ed H Oveilet. University of Ottawa Press, Ottawa

Follett BK, Scanes CG and Cunningham FJ (1972) A radioimmunoassay for avian luteinizing hormone journal of Endocrinology 52 359-378

Follett BK, Farner DS and Mattocks PW (1975) Luteinizing hormone in the plasma of white-crowned sparrows (Zonotrichia leucophrys gembelii) during artificial photostimulation General and Comparative Endocrinology 26 126-134

Foster RG, Plowman G, Goldsmith AR and Follett BK (1987) Immunohistochemical demonstration of marked changes in the LHRH system of photosensitive and photorefractory European starlings (Sturnus vulgaris) Journal of Endocrinology 115 211-220

Goldsmith AR and Nicholls T) (1984) Recovery of photosensitivity in photorefractory starlings is not prevented by testosterone treatment General and Comparative Endocrinology 56 210-217

Goldsmith AR, Ivings WE, Pearce-Kelly AS, Parry DM, Plowman DM, Nicholls TJ and Follett BK (1989) Photoperiodic control of the LHRH neurosecretory system of European starlings (Sturnus vulgaris) during puberty and the onset of photorefractoriness Journal of Endocrinology 122 255-268

Hahn TP and Ball GF (1995) Changes in brain GnRH associated with photorefractoriness in house sparrows (Passer domesticus) General and Comparative Endocrinology $99349-363$

Nicholls TJ, Goldsmith AR and Dawson A (1988) Photorefractoriness in birds and comparison with mammals Physiological Reviews 68 133-176

Parry DM, Goldsmith AR, Millar RP and Glennie LM (1997) Immunocytochemical localisation of GnRH precursor in the hypothalamus of European starlings during sexual maturation and photorefractoriness Journal of Neuroendocrinology 9 235-243

Saldanha CJ, Deviche PJ and Silver R (1994) Increased VIP and decreased GnRH expression in photorefractory dark-eyed Juncos Junco hyemalis. General and Comparative Endocrinology 93 128-136 
Sharp PJ (1996) Strategies in avian breeding cycles Animal Reproduction Science 42 505-513

Sharp PJ, Talbot RT, Main GM, Dunn IC, Fraser HM and Huskisson NS (1990) Physiological roles of chicken LHRH-I and -II in the control of gonadotrophin release in the domestic chicken Journal of Endocrinology 124 291-299
Wilson FE (1985) Androgen feedback-dependent and -independent control of photoinduced LH secretion in male tree sparrows (Spizella arborea) Journal of Endocrinology 105 141-152

Wilson FE and Donham RS (1988) Daylength and control of seasonal reproduction in male birds. In Processing of Environmental Information in Verfebrates pp 101-119 Ed MH Stetson. Springer-Verlag, New York 